\title{
System Noise Prediction of the DGEN 380 Turbofan Engine
}

Jeff Berton

NASA Glenn Research Center

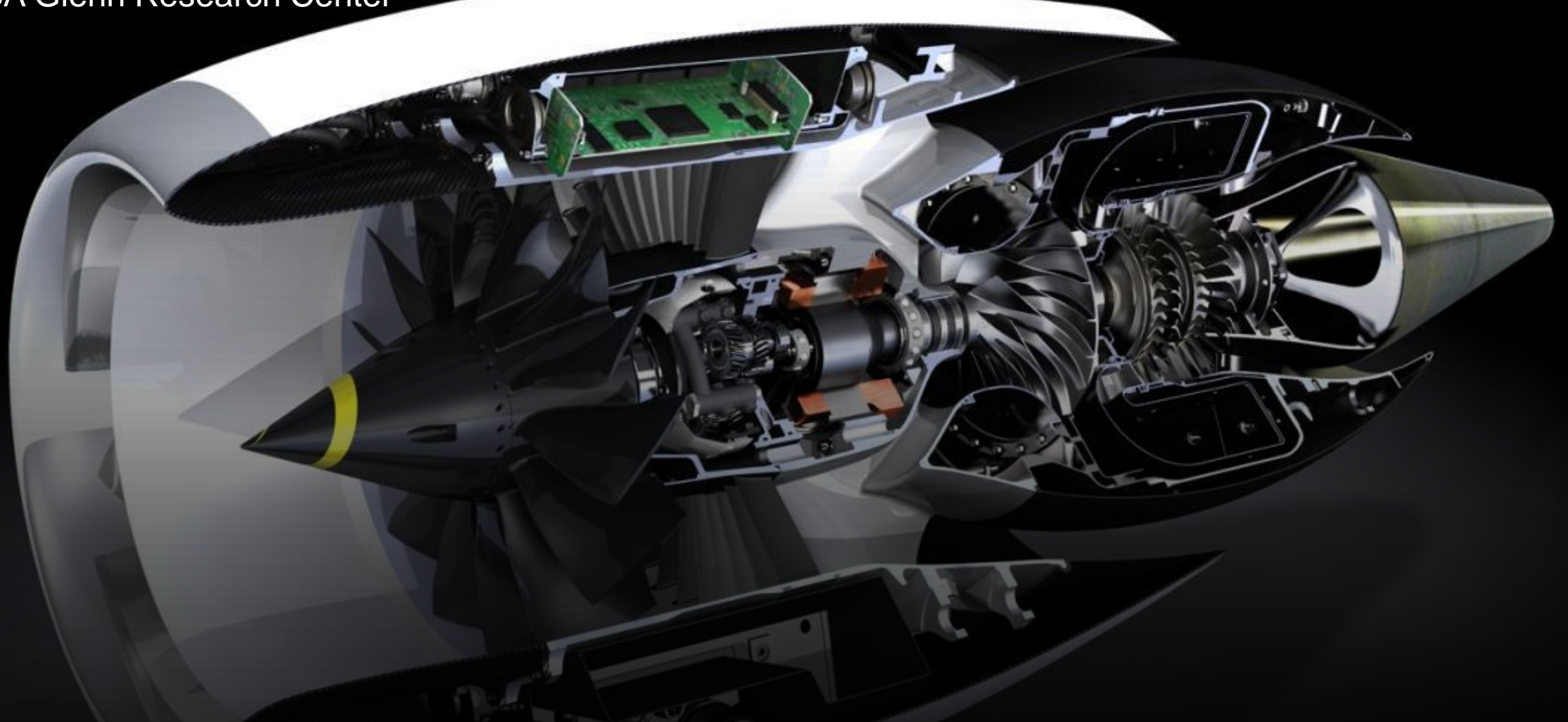

AIAA Aviation and Aeronautics Forum and Exposition

$21^{\text {st }}$ AIAA/CEAS Aeroacoustics Conference

Dallas, TX, June 22-26, 2015 


\section{Introduction}

- The DGEN 380 is a small, twinspool, separate-flow, unboosted, geared turbofan manufactured by Price Induction

- 570lb static thrust

- 14in diameter fan

- 7.6 bypass ratio

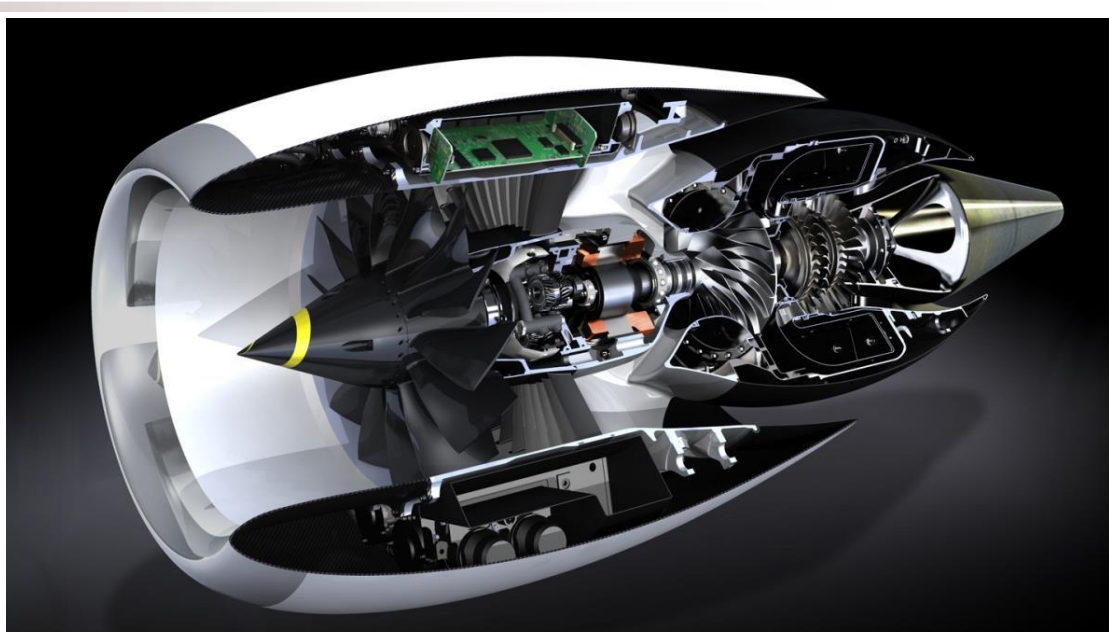

- Promoted for a small, 4- to 5-place twinjet application in the emerging personal light jet market

- Designed for aircraft operating in the regime currently dominated by propeller-driven airplanes under $25,000 \mathrm{ft}$ and $250 \mathrm{ktas}$

- DGEN engine on promotional U.S. tour in July, 2014; arrangements made for one-day acoustic test in NASA Glenn's Aero-Acoustic Laboratory dome on July 25

- NASA has interest in purchasing a DGEN to test propulsion technologies in a relevant engine environment; thus, interest in DGEN system noise 


\section{System Noise Prediction}

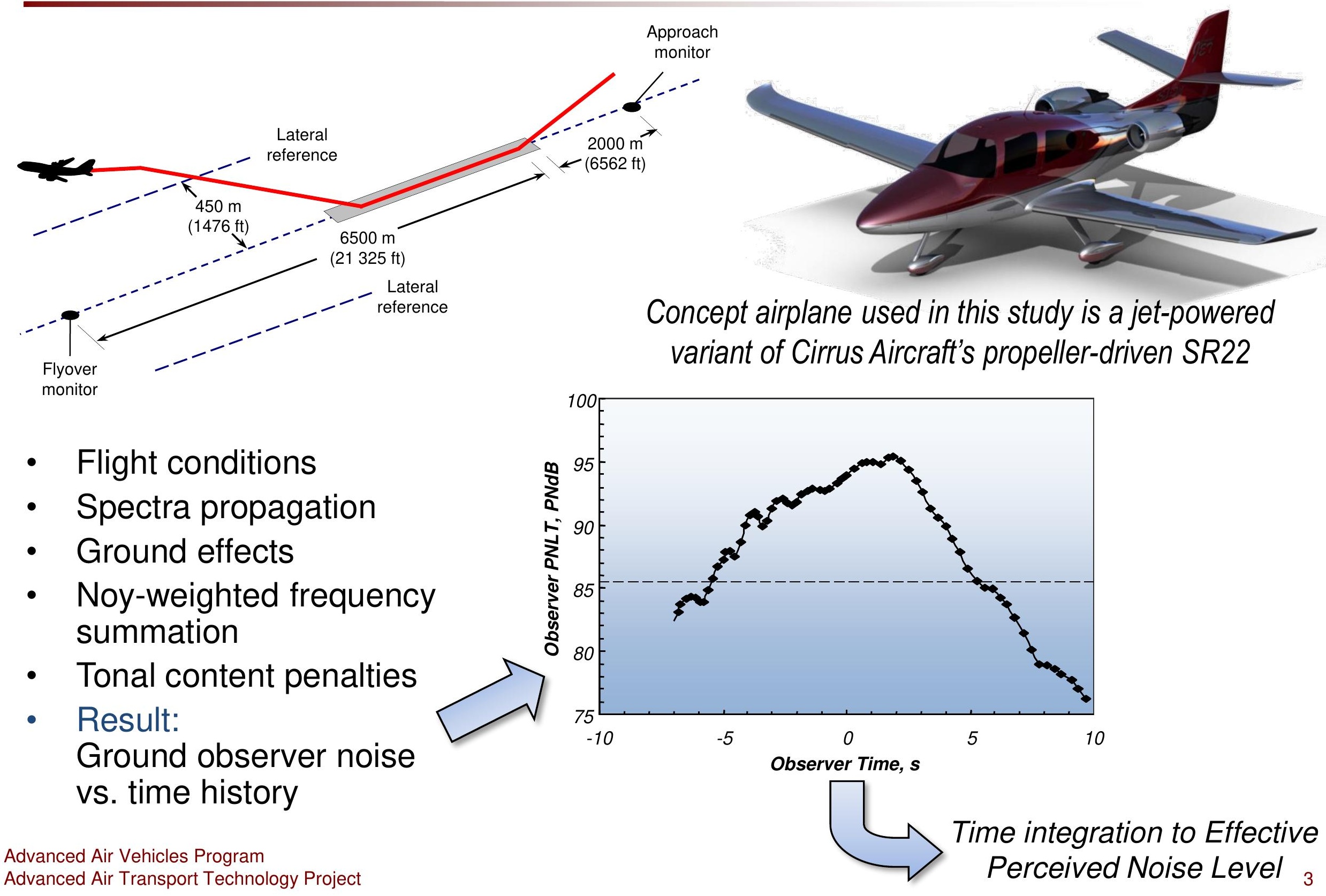




\section{Method of Analysis}

- Most expedient method for computing EPNL is to use measured engine spectra directly with a system noise analysis and propagation tool:

- Measured spectra analytically "flown" on a trajectory past ground observers

- Propagation and ground effects applied, EPNL computed for each observer

- Convection and Doppler flight effects applied to improve accuracy

- Issues with this approach:

- Engine behavior is different in flight than at ground level

- Noise measured statically on ground not wholly representative of noise in flight

- Jet mixing noise is a distributed source radiating along the axial plume of exhaust

- Approach used in this study:

- Semi-empirical noise prediction methods are derived; used in place of measured noise

- Noise surrogate models functions of engine state variables; react with flight conditions

- Surrogate models are calibrated to static spectra measured at NASA

- Physics-based models are relied on to project spectra to arbitrary flight conditions

- Surrogate models in place of actual spectra allows for removal of extraneous or spurious portions of the spectra not believed to be genuine engine noise

- Each noise source can easily be manipulated mathematically 


\section{DGEN 380 Test in NASA's Aero-Acoustic Propulsion Laboratory}

Overhead

- I microphone array $(32 \mathrm{tt} t 5 \mathrm{ft})$

$12 \mathrm{ft}$ microphone

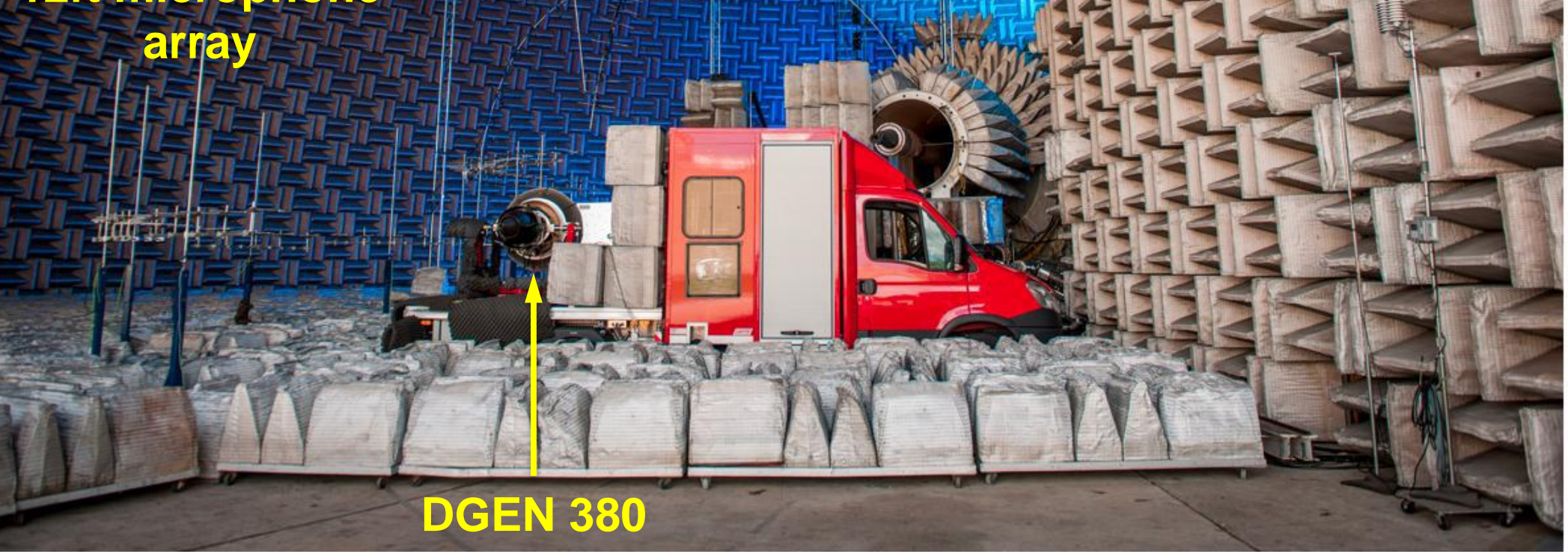




\section{Engine Source Modeling and Calibration (1)}

- One-day static engine test in NASA Glenn dome

- Six throttle settings (47\% to $96 \%$ N1max)

- 24-microphone overhead array; $32 \mathrm{ft}$ to $57 \mathrm{ft}$ radius

- Narrowband sound pressure levels collected @12.2Hz BW

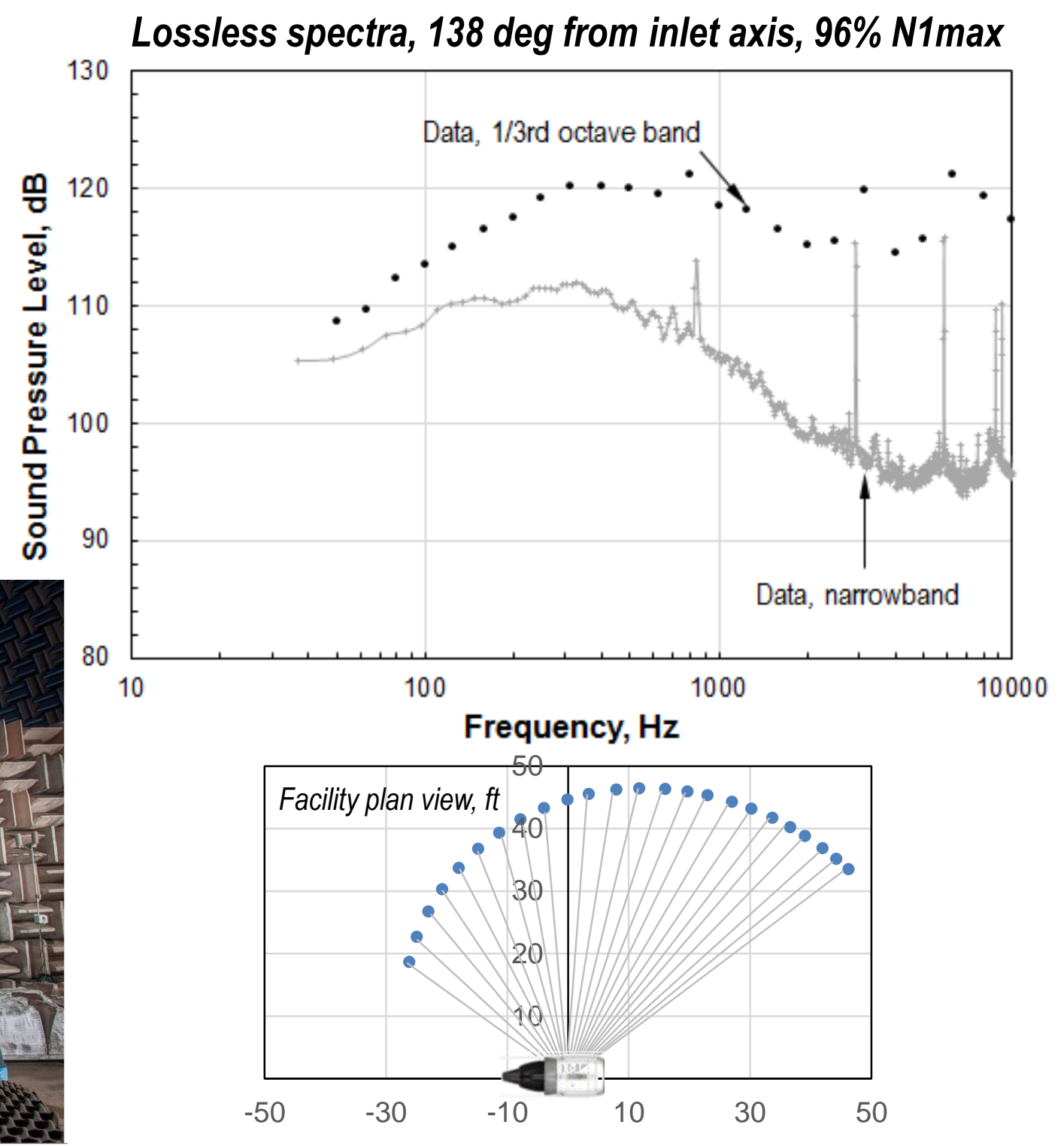




\section{Engine Source Modeling and Calibration (2)}

\section{Fan noise:}

- Based on empirical Heidmann formulation (1979), recalibrated for modern, wide-chord fans (2014)

- Acoustic power proportional to mass flow, stage temperature rise, and relative tip Mach

- Doppler and convection terms relied on to project source to flight conditions

- Calibration variables

- $x_{1}$ amplitude

- $x_{2}$ curvature

- $x_{3}-x_{6}$ discrete interaction tone levels
Lossless spectra, 138 deg from inlet axis, 96\% N1max

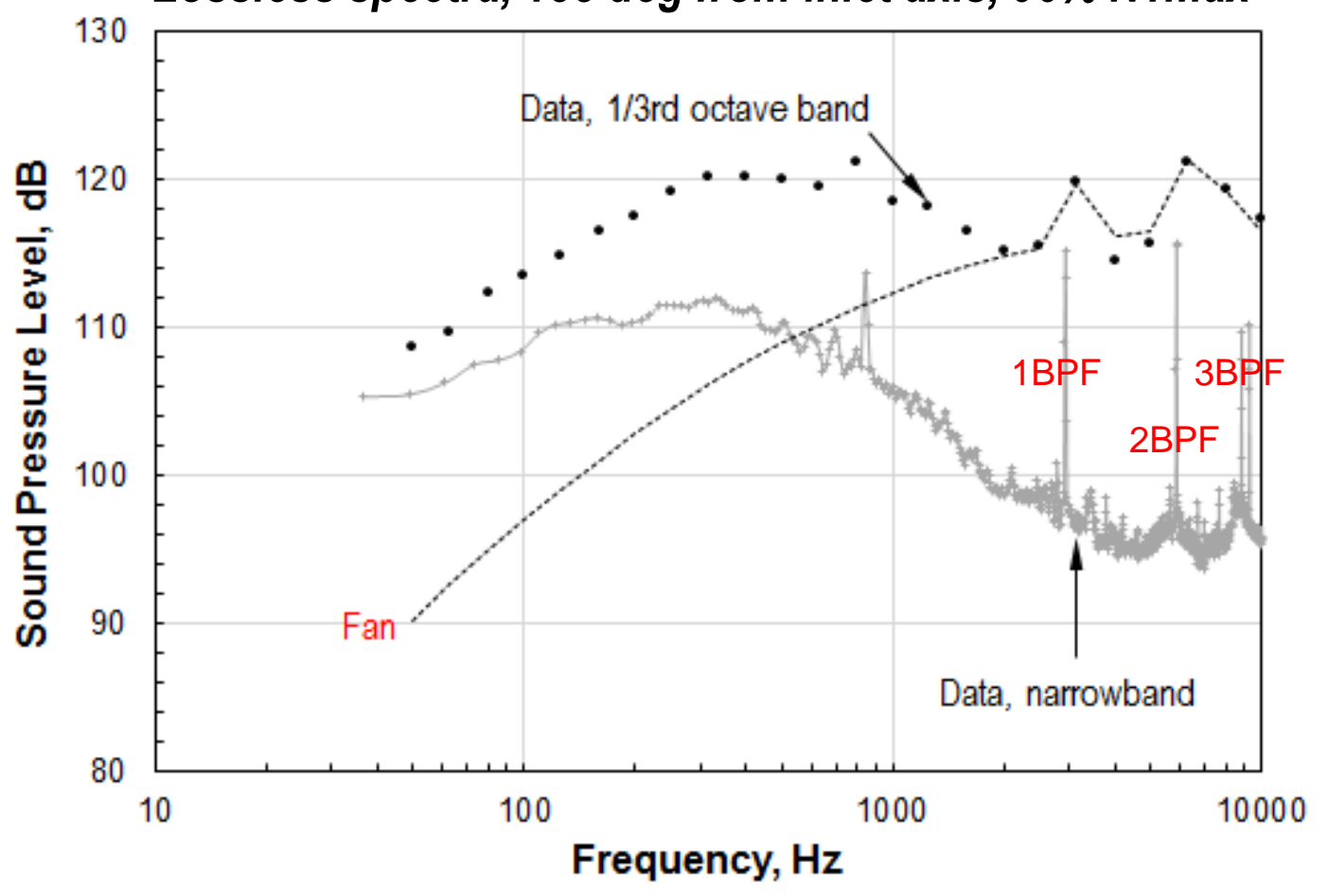

Fan noise model (after Heidmann, et al.):

$$
L_{\text {Fan }}(f, \theta)=10 \log _{10}\left\{x_{1} \frac{\dot{m}}{\dot{m}_{\text {Ref }}}\left[\frac{\Delta T_{\text {Fan }}}{T_{\text {Ref }}}\right]^{2} G\left(M_{r}\right) \frac{D(\theta) S\left(f, x_{2-6}\right)}{\left[1-M_{f} \cos \theta\right]^{k}}\right\}
$$




\section{Engine Source Modeling and Calibration (3)}

\section{Shaft noise:}

- Homebrew empirical function

- High- and low-pressure spool speeds used as independent variables

- Filtered at shaft passage frequencies

- Doppler and convection terms relied on to project source to flight conditions

- Calibration variables

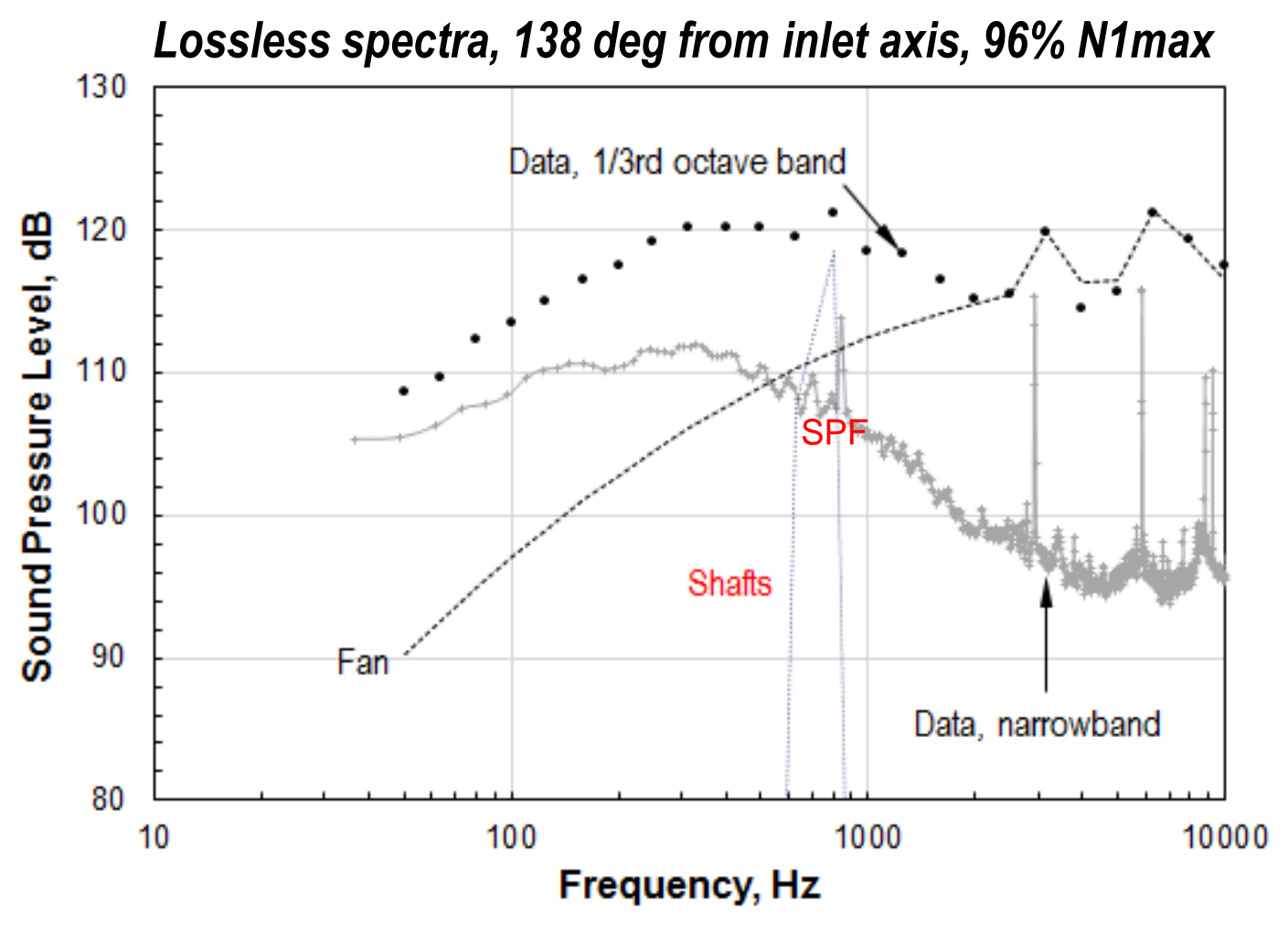

- $x_{7}$ low-spool tone

- $x_{8}$ high-spool tone

Shaft noise model:

$$
L_{\text {Shafts }}(f, \theta)=10 \log _{10}\left\{x_{7} x_{8} H\left(N_{L}, N_{H}\right) \frac{D(\theta) S(f)}{\left[1-M_{f} \cos \theta\right]^{k}}\right\}
$$




\section{Engine Source Modeling and Calibration (4)}

Core noise:

- Based on 1976 SAE method

- Acoustic power proportional to burner mass flow, temperature rise, and density

- Difficult to tell when, or if, jet noise is masquerading as core noise or vice versa

- Source signal separation coherence techniques

- Use low engine power settings as a guide

- Calibration variables

- $\quad x_{9}$ amplitude

- $x_{10}$ curvature
Lossless spectra, 138 deg from inlet axis, 96\% N1max

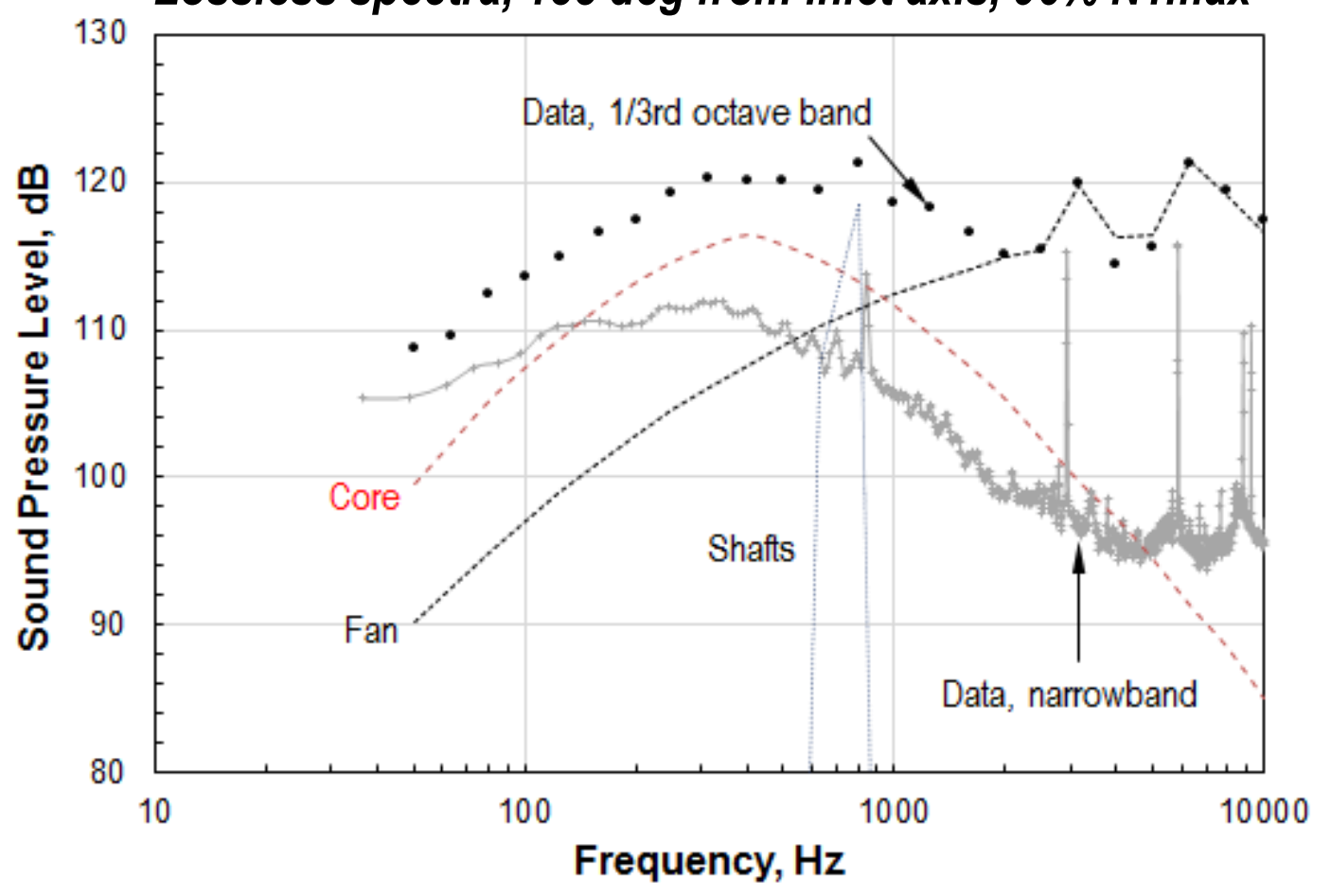

Core noise model (after Matta):

$L_{\text {Core }}(f, \theta)=10 \log _{10}\left\{x_{9} \frac{\dot{m}}{\dot{m}_{\text {Ref }}}\left[\frac{\Delta T_{\text {Comb }}}{T_{\text {Ref }}}\right]^{2}\left[\frac{\rho_{\text {Comb }}}{\rho_{\text {Ref }}}\right]^{2} \frac{D(\theta) S\left(f, x_{10}\right)}{\left[1-M_{f} \cos \theta\right]^{k}}\right\}$ 


\section{Engine Source Modeling and Calibration (5)}

\section{Jet noise:}

- Based on 2009 Stone method

- Jet mixing noise modeled as three virtual sources

- $\quad$ Each spectrum is adjusted to the microphone distance to exploit model's convection/refraction features

- Calibration variable: $x_{11}$ amplitude

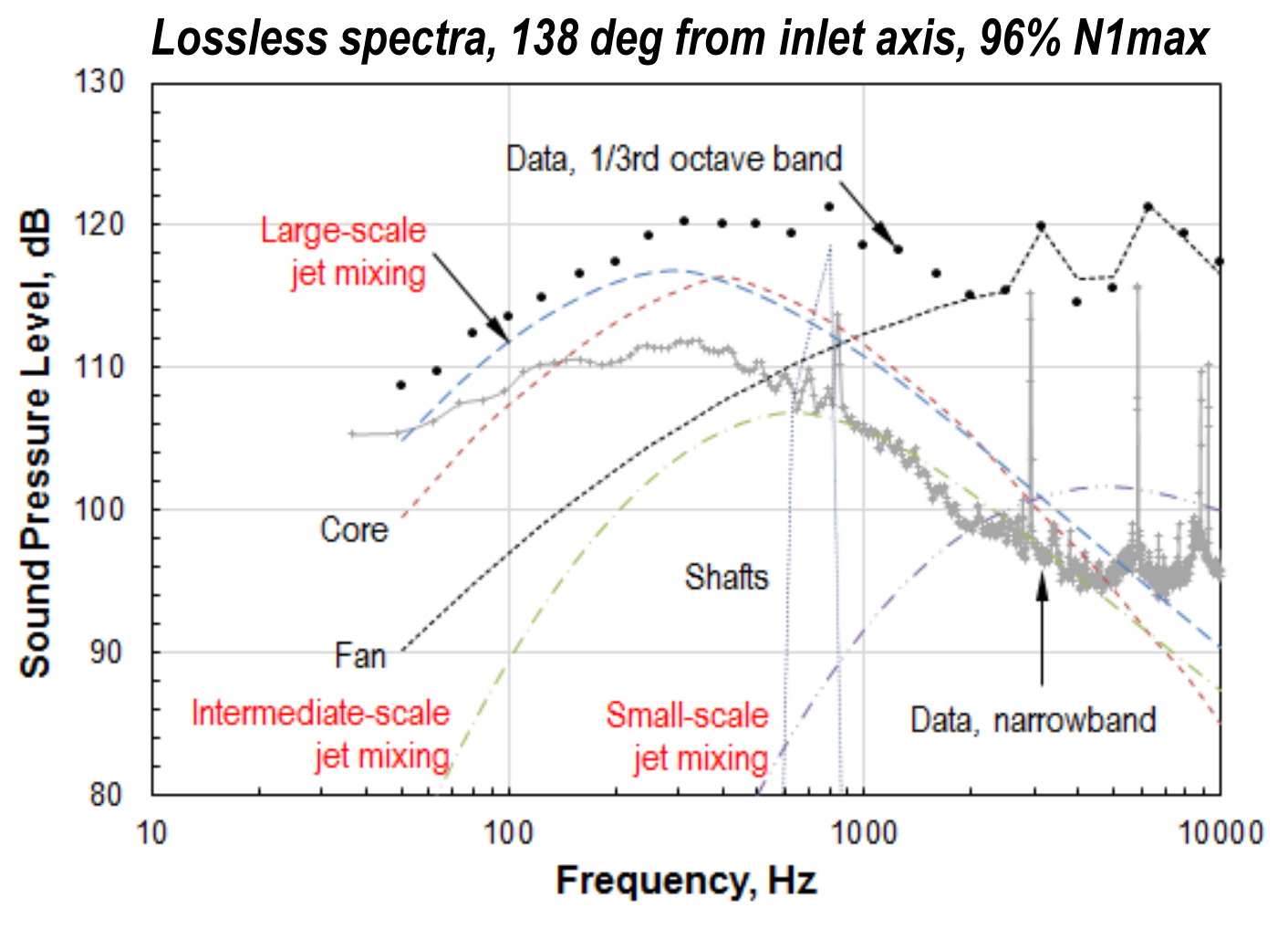

Jet noise model (after Stone):

$L_{J e t}(f, \theta)=10 \log _{10}\left\{x_{11}\left(V_{e} / c_{R e f}\right)^{n}\left(\rho / \rho_{\text {Ref }}\right)^{\omega} \frac{D\left(\theta_{e}\right) S\left(f, \theta_{e}\right)}{\left(1+M_{c} \cos \theta\right)^{2}+\alpha^{2} M_{c}^{2}}\right\}$ 


\section{Engine Source Modeling and Calibration (6)}

- Optimizer used to aid fitment of noise models to measurements

- Imperfect models, imperfect data... composite objective:

- Sound pressure levels

- Perceived noise level with tone penalty correction

- Minimum, nonzero $O(\mathbf{x})$ does not result in a unique solution

- Values of $x$ should not stray too far from their nominal values, set limits

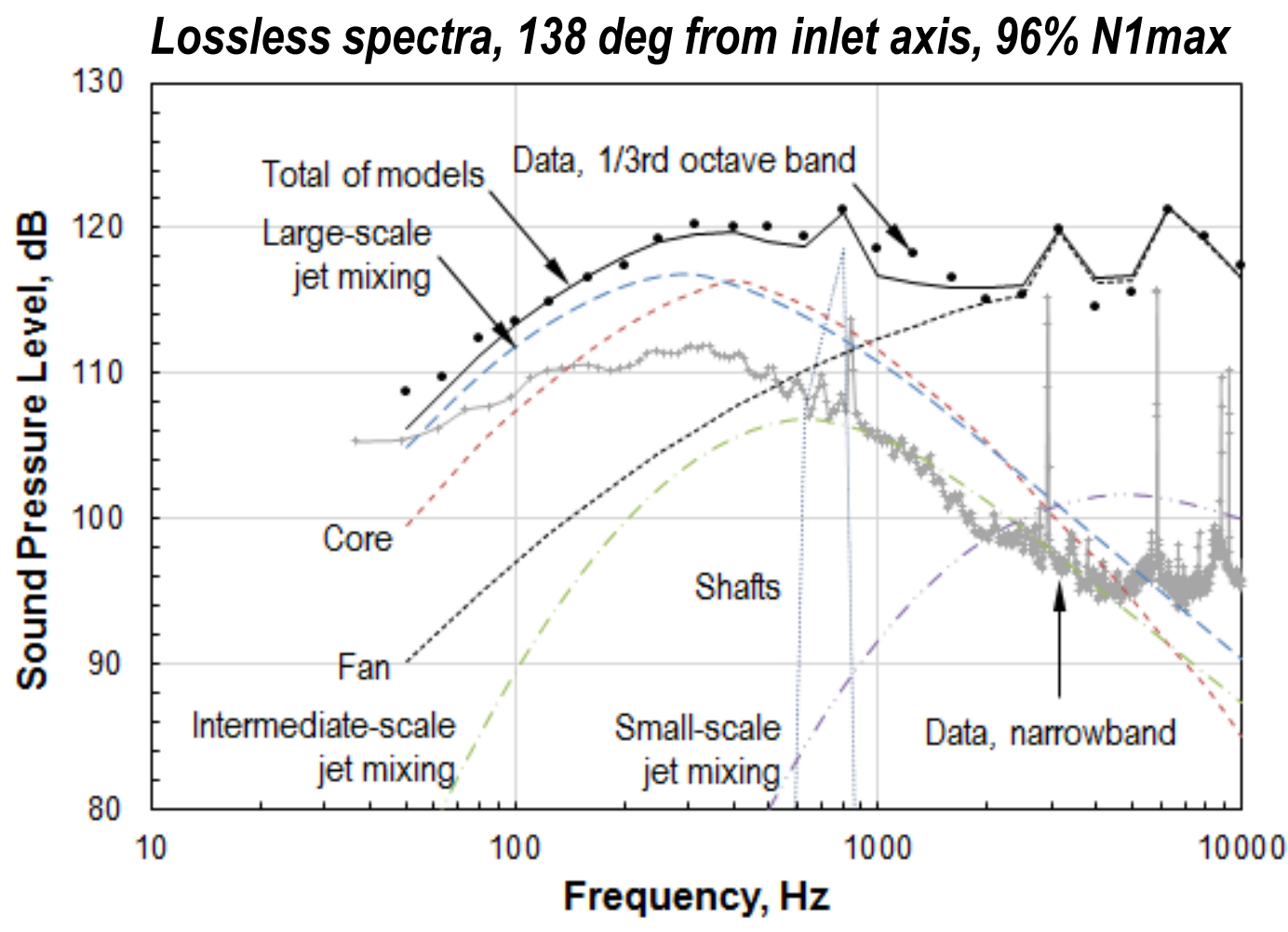




\section{Wing Planform Shielding}

- Maekawa diffraction loss method

- Implemented as function of Fresnel number

- Applied to fan and core noise sources

- Not subject to shielding:

- Airframe noise sources

- Jet noise:

A distributed source generated downstream throughout axial exhaust plume

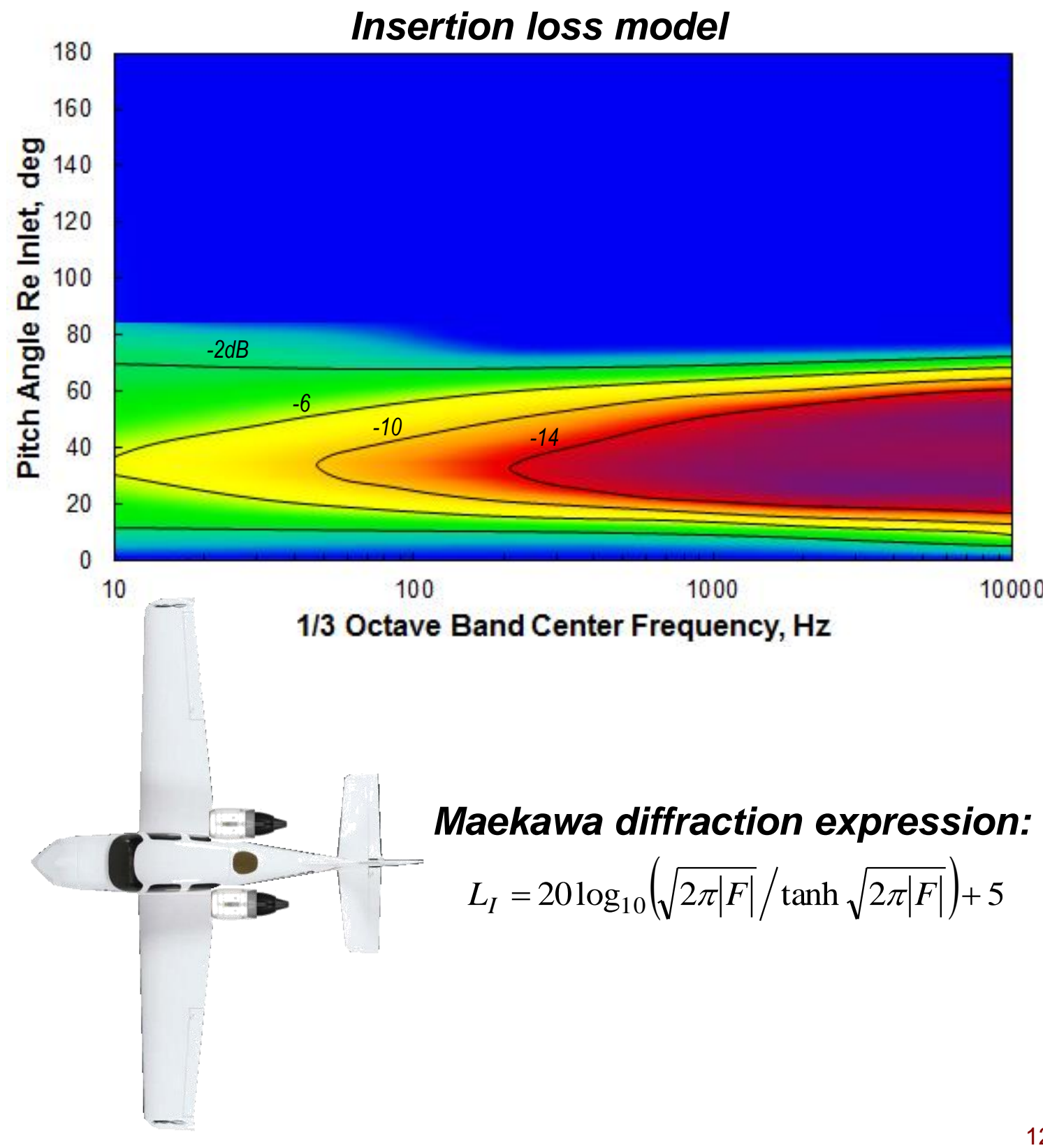




\section{Airplane Trajectory}

- Cirrus SR22 takeoff at 3400lb gross weight, $50 \%$ flaps

- Noise abatement power cutback; climb gradient:

- $4 \%$, all engines operating

- Zero, one engine inoperative

- Approach at 2790lb

- Three-degree approach glide slope, with flaps fully extended, gear down
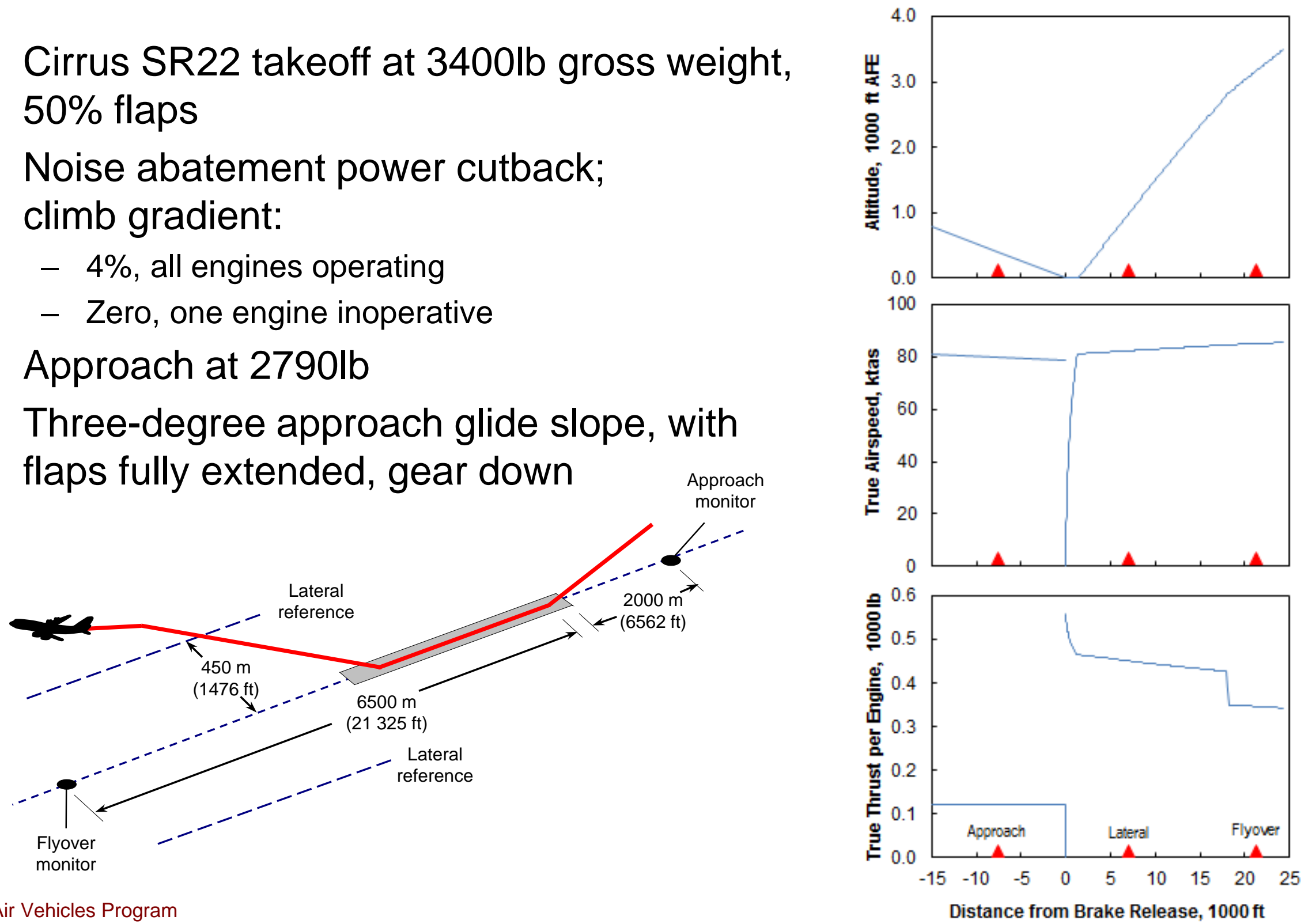


\section{Noise Prediction Results}

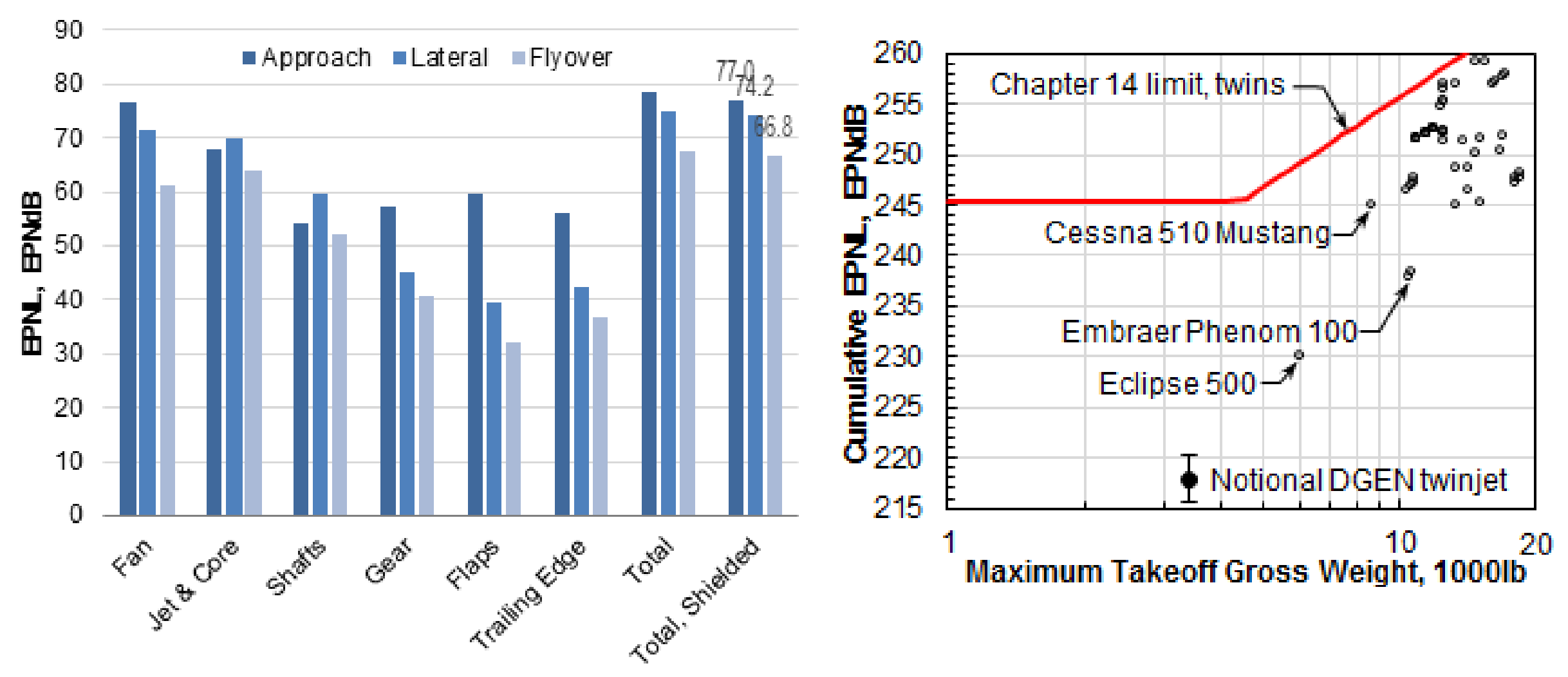

- Chapter 4 cumulative margin: $\quad 53.1 \mathrm{EPNdB}$

- Chapter 14 cumulative margin: 27.4 EPNdB

Meets NASA's "N+3" noise goal, albeit at a much smaller size! 


\section{Monte Carlo Uncertainty Analysis}

- Real engine, notional airplane... Uncertainty analysis needed!

- Modeling unknowns chosen by top-down decomposition of problem

- Variables categorized into trajectory, source levels, environmental \& installation classes

- Variables chosen to represent effects that would cause values to stray from benchmark during airplane development

- Benchmark noise model transformed into stochastic model

- Variables randomly permuted around

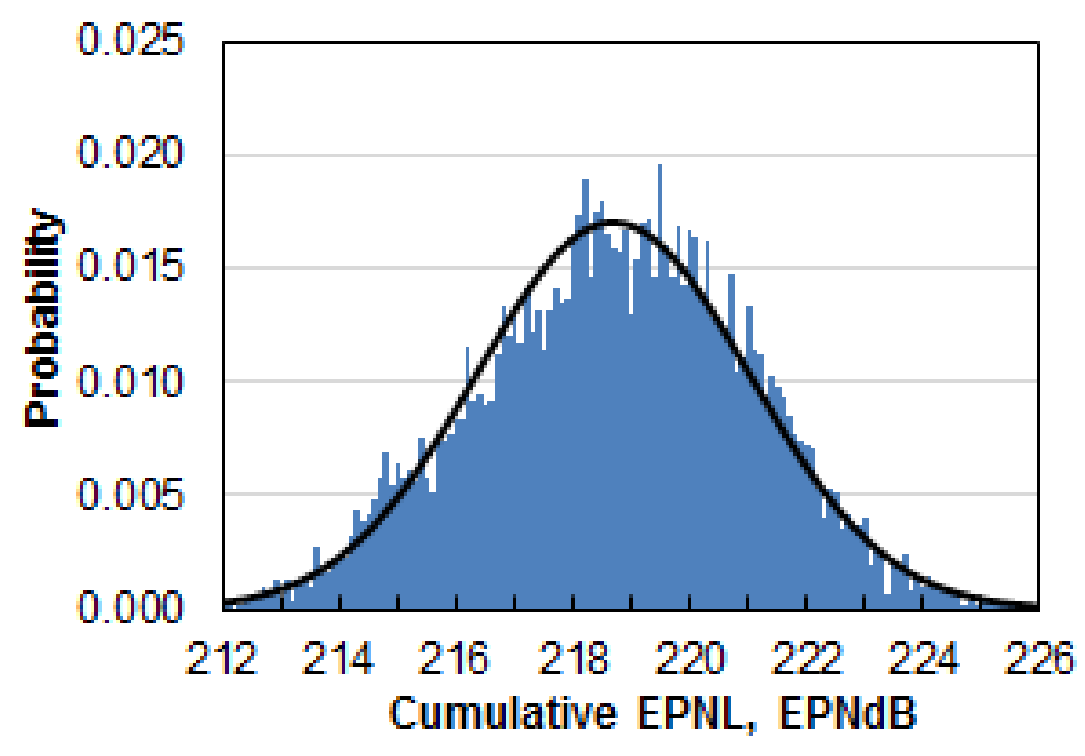

Histogram and normal distribution generated from 8000 samples (bin span 0.1 EPNdB) benchmark case

\begin{tabular}{l|c|c|c|c}
\hline \hline Statistic & Approach & Lateral & Flyover & Cumulative \\
\hline Benchmark case & 77.0 & 74.2 & 66.8 & 217.9 \\
Minimum of samples & 74.3 & 70.6 & 64.4 & 209.5 \\
Maximum of samples & 80.5 & 78.1 & 69.7 & 226.4 \\
Range of samples & 6.2 & 7.6 & 5.3 & 17.0 \\
Mean of samples & 77.3 & 74.6 & 66.8 & 218.7 \\
Standard deviation & 0.9 & 1.2 & 0.8 & 2.3 \\
\hline \hline
\end{tabular}




\section{Summary}

- Static noise measurements of a Price Induction DGEN 380 turbofan were collected at NASA Glenn Research Center

- Noise source models were calibrated and used to analytically project static spectra to flight conditions

- Embedded physics-based behavior allows noise source models to react properly to changing engine state and flight conditions

- The DGEN is a quiet turbofan, owing not only to its small size, but also to its design

- Cumulative margins to Chapter 14 and Chapter 4 limits are predicted to be 27.4 and 53.1 EPNdB, respectively 


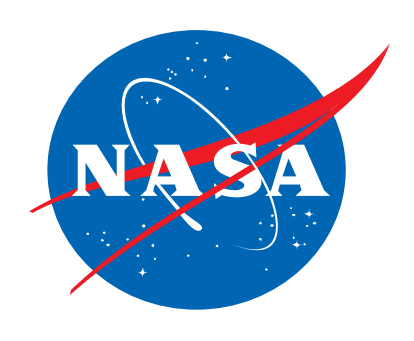




\section{Backup Slides}




\section{Simulated Engine Cycle Data}

- Empirical noise models require engine cycle data for noise level scaling

- Engine cycle data not measured during acoustic test

- Price Induction's “Virtual Engine Test Bench," a DGEN 380 digital engine control unit

- Engine data response surfaces generated for steady pressures, temperatures and airflows $\left(\mathrm{ISA}+18^{\circ} \mathrm{F}\right)$ at all major engine flowstations as function of airspeed, altitude and low-spool shaft speed
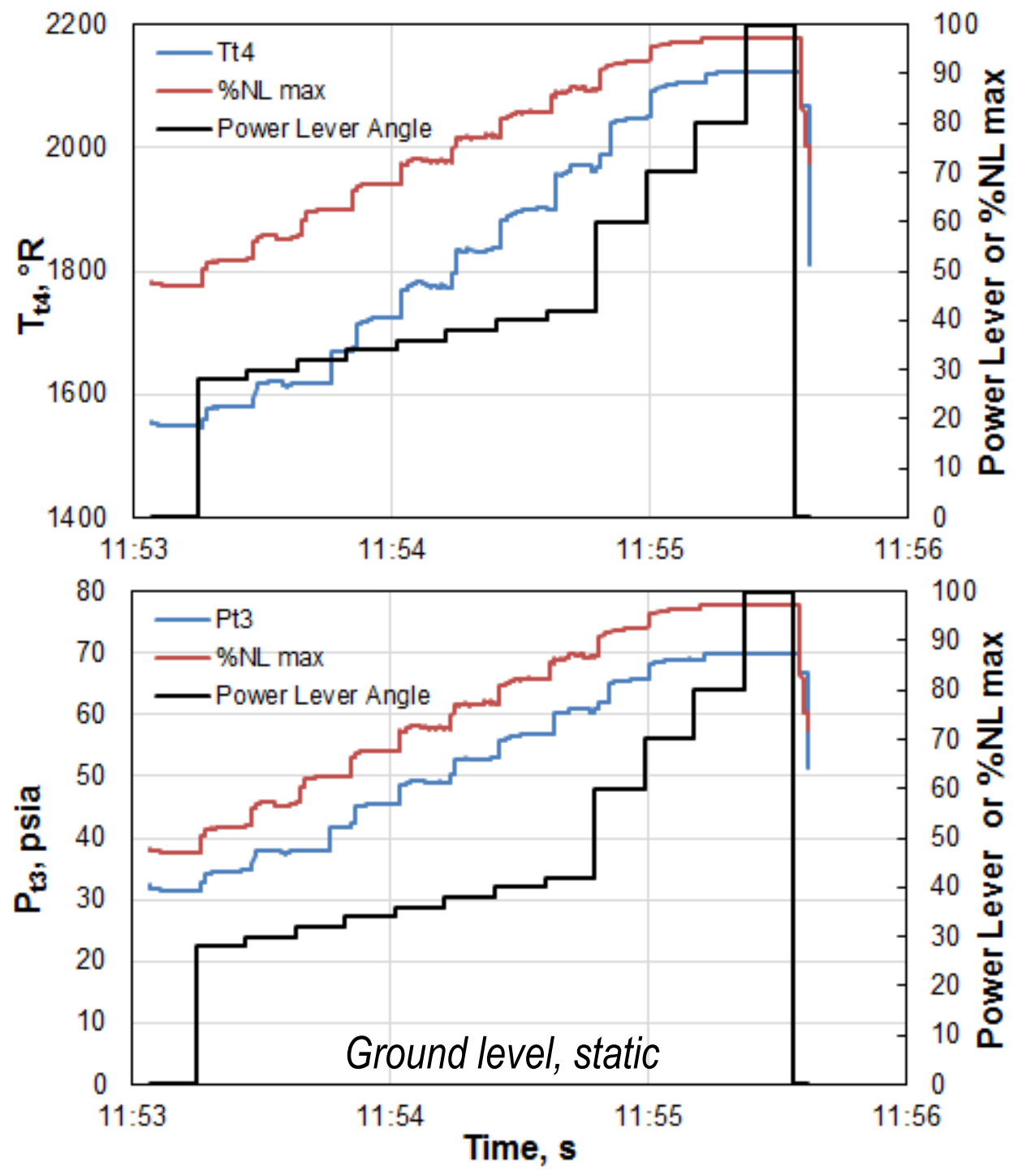


\section{Noise Prediction Results}
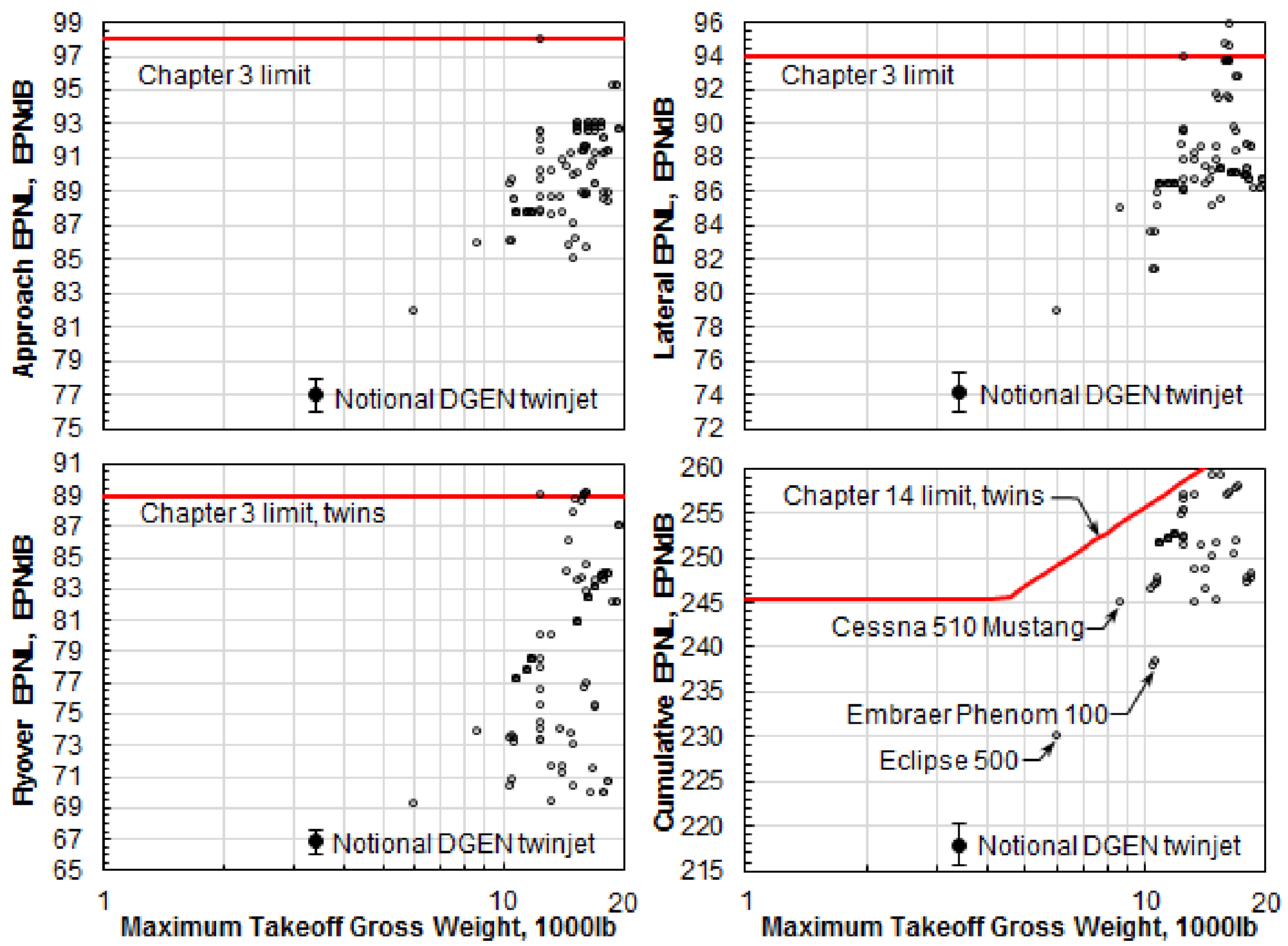


\section{Monte Carlo Uncertainty Analysis}

\section{Variables perturbed in Monte Carlo experiment}

\begin{tabular}{|c|c|c|c|c|c|c|}
\hline & Variable & Mode & Model & Min & Max & Std. Dev. \\
\hline \multirow{10}{*}{$\begin{array}{l}\text { Trajectory- } \\
\text { related effects }\end{array}$} & Approach flight Mach no. & 0.119 & Triangular & 0.112 & 0.126 & - \\
\hline & Lateral flight Mach no. & 0.123 & Triangular & 0.119 & 0.127 & - \\
\hline & Flyover flight Mach no. & 0.128 & Triangular & 0.120 & 0.150 & - \\
\hline & Approach $N_{L}$ setpoint & $60 \%$ & Triangular & $58 \%$ & $62 \%$ & - \\
\hline & Lateral $N_{L}$ setpoint & $96 \%$ & Triangular & $94 \%$ & $100 \%$ & - \\
\hline & Flyover $N_{L}$ setpoint & $90 \%$ & Triangular & $87 \%$ & $93 \%$ & - \\
\hline & Approach angle of attack & $6^{\circ}$ & Triangular & $5^{\circ}$ & $7^{\circ}$ & - \\
\hline & Lateral angle of attack & $6^{\circ}$ & Triangular & $5^{\circ}$ & $7^{\circ}$ & - \\
\hline & Flyover angle of attack & $6^{\circ}$ & Triangular & $5^{\circ}$ & $7^{\circ}$ & - \\
\hline & Flyover altitude & $3170 \mathrm{ft}$ & Triangular & $2850 \mathrm{ft}$ & $3490 \mathrm{ft}$ & - \\
\hline \multirow{7}{*}{ Source levels } & Fan noise adjustment & 0 & Normal & - & - & $1.0 \mathrm{~dB}$ \\
\hline & Core noise adjustment & 0 & Normal & - & - & $1.0 \mathrm{~dB}$ \\
\hline & Shaft noise adjustment & 0 & Normal & - & - & $1.0 \mathrm{~dB}$ \\
\hline & Jet noise adjustment & 0 & Normal & - & - & $1.0 \mathrm{~dB}$ \\
\hline & Landing gear noise adjustment & 0 & Normal & - & - & $1.5 \mathrm{~dB}$ \\
\hline & Flap noise adjustment & 0 & Normal & - & - & $1.5 \mathrm{~dB}$ \\
\hline & Trailing edge noise adjustment & 0 & Normal & - & - & $1.5 \mathrm{~dB}$ \\
\hline \multirow{3}{*}{$\begin{array}{l}\text { Environment } \\
\text { \& installation }\end{array}$} & Ground specific flow resistance & $291 \mathrm{sl} / \mathrm{s}-\mathrm{ft}^{3}$ & Triangular & $233 \mathrm{sl} / \mathrm{s}-\mathrm{ft}^{3}$ & $349 \mathrm{sl} / \mathrm{s}-\mathrm{ft}^{3}$ & - \\
\hline & Lateral attenuation adjustment & 0 & Triangular & $-2 \mathrm{~dB}$ & $2 \mathrm{~dB}$ & - \\
\hline & Wing area (shielding) & $155 \mathrm{ft}^{2}$ & Uniform & 0 & $200 \mathrm{ft}^{2}$ & - \\
\hline
\end{tabular}

